\title{
SOIL CAPABILITY AND SUITABILITY ASSESSMENT OF TUSHKA AREA, EGYPT BY USING DIFFERENT PROGRAMS (ASLE, MICROLEIS AND MODIFIED STORIE INDEX)
}

\author{
Salah Hassanien Abd El-Aziz \\ Department of Soils \& Water, Collage of Agriculture, Assiut University, Assiut, Egypt. \\ *Corresponding Author Email: small.hearts@yahoo.com
}

This is an open access article distributed under the Creative Commons Attribution License, which permits unrestricted use, distribution, and reproduction in any medium, provided the original work is properly cited.

\section{ARTICLE DETAILS}

\section{Article History:}

Received 12 November 2017 Accepted 12 December 2017 Available online 1 January 2018

\begin{abstract}
The present study was undertaken to identify the morphological, physical and chemical characteristics of soils in Tushka, Aswan governorate, Egypt, in order to classify and evaluate them from the agricultural use view point. Tushka area is located in the western desert, upper Egypt. It lies between latitudes of $22^{\circ} 48^{\prime} 00.7^{\prime \prime}$ and $22^{\circ} 28^{\prime}$ $44.2^{\prime \prime} \mathrm{N}$ and longitudes of $31^{\circ} 28^{\prime} 07.2^{\prime \prime}$ and $31^{\circ} 29^{\prime} 08.2^{\prime \prime} \mathrm{E}$. The soils of the study area were none to slightly saline (ECe ranged from 0.53 to $6.85 \mathrm{dSm}-1$ ). Soil texture was mostly sand, loamy sand and sandy loam. Soil reaction $(\mathrm{pH})$ tended to be mildly to moderately alkaline with a range of 7.6 to 8.1. Calcium carbonate and gypsum contents were very low. The soils were classified as Typic Torripsamments, Typic Torriorthents and Lithic Torriorthents. Most of the soils understudy were suitable for agricultural use. The results revealed that the capability of soils according to ASLE program was good (C2) and fair suitable (C3), moderate suitable (S3) using MicroLEIS (Cervatana model) and good, fair and poor using Modified Storie Index. Most of the selected crops were found to be the best grown ones on soils of the S2 and S3 suitability classes by ASLE program. Also, most of the selected crops were moderately (S3) and marginally suitable (S4) by MicroLEIS-ALMAGRA model. The main limitation factors of the study area for crop production were soil texture and soil depth.
\end{abstract}

\section{KEYWORDS}

Tushka, ASLE program, MicroLEIS, Modified Storie Index

\section{INTRODUCTION}

Egypt has an arid land with almost $96 \%$ of uninhabited parts of its territory. More than ninety million inhabitants are concentrated mainly in the Nile delta and valley as well as in the northern coastal zone along the Mediterranean Sea and in small areas of Western desert where lands are suitable for agricultural production $[1,2]$.

The main challenge facing Egypt today is the need for better development and management of natural resources to meet the growing needs of the nation. The ratio between land and human resources is the most important problem in Egypt [3]. The horizontal agricultural expansion in the Western desert is one of the most important objectives of Egyptian agricultural policy to meet the food security needs of the growing population [4]. The agricultural expansion in new desert areas is also a priority to compensate the successive loss of agricultural land in Egypt [5].

Southwest Tushka area which lies south west of Egypt is considered as one of the promising areas for agricultural expansion and development [6]. Land assessment allows lands to be evaluated for agricultural use in accordance with their physical and chemical capacities as well as limitations to protect soil resources from degradation during potentialities achieving farmers' demands for optimal crop production [7]. Since wheat, barley, maize and sorghum are strategic crops in Egypt and most farmers devote high surface areas to grow wheat each year, these crops were selected to be evaluated for soil adequacy assessment of this area.

The general view of geology and geomorphology of the western desert, which includes the area understudy [8]. Essentially it is a desertic plateau with a vast flat expansion of rocky ground or numerous closed depressions. The greatest altitude is attained in the extreme south western corner where the general plateau character is disturbed by the great mountain of Gebel Uweinat. North of this mountain, a broad high terrain plateau, known as Gilf El-Kebir, extends for more than $200 \mathrm{~km}$. This sandstone plateau is bordered in the south by a prominent escarpment, that descends gradually to the north and east directions forming a very extensive pediment sandy plain. This sandy plain is dotted in several parts by many rock exposures of Tertiary volcanic volcanic origin and basement complex rocks of granitites. Cretaceous rocks formed of what is called the Nubian formation, which is essentially sandstone, occupy the sand plain. In general, soil characteristics, classification and evaluation of some parts in Egypt using different programs (ASLE, MicroLEIS and Modified Storie Index) which were studied at regional stages were investigated by many researchers [9-22]. The main objective of this research is to evaluate and compare the land suitability of Tushka area, Egypt for some principal crops using different evaluation systems. Several crops were selected to assess their convenience to be grown in the studied area. This study is needed to get useful information about these soils. It would help agricultural investment of various parts of Tushka area.

\section{MATERIAL AND METHODS}

\subsection{Field Description and Soil Sampling}

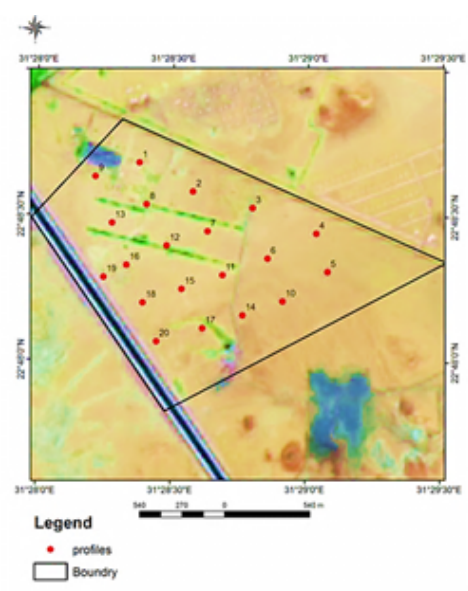

Figure 1: The soil profile location map of the study area

The area under investigation is located on the east side of Abu-Simbel/ Aswan road which is ( $\mathrm{km} \mathrm{50)}$ north of Abu-Simbel city. It is a part of the western desert plateau and lies between latitudes $22^{\circ} 48^{\prime} 00.7^{\prime \prime}$ and $22^{\circ}$ 
$48^{\prime} 00.7^{\prime \prime}$ and $22^{\circ} 28^{\prime} 44.2^{\prime \prime} \mathrm{N}$ and longitudes $31^{\circ} 28^{\prime} 07.2^{\prime \prime}$ and $31^{\circ} 29^{\prime}$ $08.2^{\prime \prime}$ E (Figure 1). Twenty soil profiles were selected to represent the area under investigation according to the geological, topographic and recent aerial photographic maps of the study area. The profiles were dug down to parent rock and described for their morphological characteristics according to the standard procedures [23-25]. Soil samples were collected from profile layers according to the vertical morphological variations. The samples were air-dried, crushed, passed through a $2 \mathrm{~mm}$ sieve, and kept for different analysis. Soil color of both dry and moist samples was determined using Munsell color chart was determined [26]. The study also exploited the use of geographic information systems (GIS, ArcView, 10) for mapping the soils of the study area.

\subsection{Climate of the Study Area}

The most important climate characteristics necessary for the suitability determination (temperature, rainfall, relative humidity, etc.) were collected from Aswan metrological station. The study area has a mean annual rainfall of $1 \mathrm{~mm} /$ year that is concentrated in the winter season, with mean relative humidity of $9.4 \%$ and a mean annual temperature of $26.3^{\circ} \mathrm{C}$ (mean maximum temperature is $33.9^{\circ} \mathrm{C}$ and the mean minimum temperature is $18.8^{\circ} \mathrm{C}$ )

\subsection{Laboratory Analysis}

The gravels content was measured by volume according to a study [27] Particle-size distribution of the studied soils was performed according to one study by a group researcher [28]. Soil reaction $(\mathrm{pH})$ of 1:1 soil to water suspension was measured using a glass electrode [29]. Total Calcium carbonate (CaCO3) was determined by Collin's calcimeter [30, 31]. The electrical conductivity (ECe) of the solution soil paste extract was assessed by methods described in some studies [32]. Determination of soil gypsum content was done in using a graph showing the relation between the concentration and electrical conductivity of gypsum solution [30]. The exchangeable sodium percentage (ESP) of the soil samples was determined according to some research paper [32] using ammonium acetate method. The cation exchangeable capacity was measured by sodium oxalate method [33, 34].

\subsection{Soil Classification}

The dominant soil moisture regime is aridic (torric) with a hyperthermic soil temperature regime. The soils were classified up to the sub group according to Soil Taxonomy [25]. The results obtained from the visual interpretation and digital elevation model as well as field data were incorporated using GIS in order to produce the soil map of the study area.

\subsection{Land Evaluation Methods}

The studied soils were evaluated for land capability and suitability using several systems as follow:

\section{a) Land capability classification}

- Modified Storie Index Rating, [35]: The calculation was run and marked using Visual Basic for application under Microsoft Excel [36],

- MicroLEIS [37], Internet-based program, and

- Applied System of Land Evaluation (ASLE) program [38].

b) Land suitability classification.

- MicroLEIS [39], Internet-based program, and

- Applied System of Land Evaluation (ASLE) program [38].

\section{RESULTS AND DISCUSSION}

\subsection{Main Morphological Aspects of the Studied Soils}

The main morphological aspects of the studied soil profiles are shown in Table 1. The field description revealed that the topography of the landscape was almost flat to gently sloping. The elevation ranged between 192 and $208 \mathrm{~m}$ above sea level. Most of soil profiles were fairly well drained and the water table was deep $(>200 \mathrm{~cm})$. Thus, the crop growth was not affected. The dominant soil color in the studied soil profiles was reddish yellow (5YR 7/6, dry) to yellowish red (5YR 4/6, moist) or reddish yellow (7.5YR 7/6, dry) to strong brown (7.5YR 5/6, moist). However, very pale brown (10YR 8/4, dry) to yellowish brown (10YR 5/6, moist) colors were also detected. This could possibly be attributed to the heterogeneity of parent materials and/or multidepositional regime. No effervescence with dilute $\mathrm{HCl}$ was observed in all pedons indicating absence of CaCO3.The soil structure of most soil profiles was platy and subangular blocky; the consistence was slightly hard to extremely hard (dry) and loose to friable (moist). The area was virgin without any natural vegetation. The horizon boundaries were abrupt in distinctness and smooth to wavy in topography.

Table 1: The main morphological aspects of the studied soil profiles

\begin{tabular}{|c|c|c|c|c|c|c|c|c|c|c|c|c|c|c|}
\hline \multirow{2}{*}{ 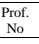 } & \multirow{2}{*}{$\begin{array}{l}\text { Elevation } \\
\text { A.S.L. }(\mathrm{m})\end{array}$} & \multirow{2}{*}{ Horizon } & \multirow{2}{*}{$\begin{array}{l}\begin{array}{l}\text { Depth } \\
(\mathrm{cm})\end{array} \\
\end{array}$} & \multicolumn{3}{|c|}{\begin{tabular}{|l} 
Soil Color \\
\end{tabular}} & \multirow{2}{*}{ Gravel } & \multirow{2}{*}{$\begin{array}{l}\text { Texture } \\
\text { (I) } \\
\end{array}$} & \multicolumn{3}{|c|}{ Soil Structure (II) } & \multicolumn{2}{|c|}{ Consistence (III) } & \multirow{2}{*}{$\begin{array}{c}\text { Boundary } \\
\text { (IV) }\end{array}$} \\
\hline & & & & Hue & Dry & Moist & & & Grade & Size & Type & & & \\
\hline 1 & 205 & $\mathrm{Cl}_{2 \mathrm{C}}$ & $0-20$ & 10YR & $8 / 4$ & $5 / 4$ & few & LS & $\frac{1}{2}$ & ${ }^{f}$ & $\mathrm{pl}$ & sh & $\begin{array}{ll}\text { loose } \\
\text { friable }\end{array}$ & \\
\hline & & ${ }_{\mathrm{C} 2}^{2 \mathrm{C} 2}$ & $\begin{array}{l}20-100 \\
0.25\end{array}$ & $\begin{array}{l}5 \mathrm{SR} \\
10 Y \mathrm{YR}\end{array}$ & $\begin{array}{l}4 / 4 \\
8 / 4\end{array}$ & $\begin{array}{l}5 / 6 \\
5 / 6\end{array}$ & & $\begin{array}{l}\text { SL } \\
\text { LS }\end{array}$ & & $\mathrm{c}$ & $\mathrm{pl}$ & vh & friable & \\
\hline 2 & 198 & & $25-50$ & & 8/4 & $7 / 4$ & - & s & 2 & $\mathrm{~m}$ & & $\mathrm{~h}$ & $\begin{array}{l}\text { loose } \\
\text { loose }\end{array}$ & $\begin{array}{l}\text { as } \\
\text { aw }\end{array}$ \\
\hline & & ${ }_{2 \mathrm{C} 3}$ & $50-100$ & & $6 / 6$ & $5 / 6$ & - & s & 2 & & $\mathrm{pl}$ & vh & friable & \\
\hline 3 & & $\mathrm{Cl}$ & $0-15$ & 7.5YR & $7 / 4$ & $5 / 6$ & - & $\mathrm{s}$ & 1 & $\mathrm{f}$ & $\mathrm{pl}$ & sh & loose & as \\
\hline & 193 & $2 \mathrm{C} 2$ & 15-70 & 7.5YR & 6/4 & 5/6 & & LS & 1 & $\mathrm{~m}$ & sbk & $\mathrm{h}$ & loose & \\
\hline 4 & 193 & ${ }_{\mathrm{R}}^{\mathrm{C} 1}$ & $\begin{array}{c}0-20 \\
20-50\end{array}$ & $\begin{array}{l}10 \mathrm{YR} \\
10 \mathrm{YR}\end{array}$ & $8 / 4$ & $\begin{array}{l}5 / 6 \\
5 / 6\end{array}$ & few & $\begin{array}{l}\text { SL } \\
\text { SL }\end{array}$ & 2 & $\mathrm{~m}$ & sl & $\begin{array}{l}\text { so } \\
\text { vh }\end{array}$ & $\begin{array}{l}\text { loose } \\
\text { friable }\end{array}$ & as \\
\hline & & & & $7.5 \mathrm{YR}$ & $6 / 5$ & $4 / 4$ & - & SL & & & sl & so & Loose & \\
\hline 5 & 195 & $2 \mathrm{C} 2$ & $15-30$ & $10 \mathrm{YR}$ & $8 / 4$ & $5 / 6$ & & LS & 1 & $\mathrm{f}$ & $\mathrm{pl}$ & sh & v. friable & aw \\
\hline & & 3C3 & $\begin{array}{l}30-90 \\
0.20\end{array}$ & $\begin{array}{l}7.5 \mathrm{YR} \\
5 \mathrm{YR}\end{array}$ & 5/6 & ${ }_{4 / 6}^{44 /}$ & & $\begin{array}{l}\text { SL } \\
\text { SL }\end{array}$ & 2 & $\mathrm{f}$ & sbk & $\mathrm{h}$ & $\begin{array}{l}\text { friable } \\
\text { loose }\end{array}$ & as \\
\hline 6 & 192 & $\begin{array}{c}\mathrm{C}_{2} \\
-\end{array}$ & & $\begin{array}{l}5 \mathrm{YR} \\
5 \mathrm{YR}\end{array}$ & $7 / 6$ & 4/6 & few & SL & 1 & $f$ & $\begin{array}{c}\text { st } \\
\text { sbk }\end{array}$ & $\begin{array}{c}\text { so } \\
\text { h }\end{array}$ & $\begin{array}{l}\text { loose } \\
\text { v. friable }\end{array}$ & $\begin{array}{l}\text { as } \\
\text { as }\end{array}$ \\
\hline & & $\mathrm{C}_{3}$ & $50-100$ & $5 \mathrm{YR}$ & $7 / 6$ & $4 / 6$ & - & SL & 2 & $\mathrm{f}$ & $\mathrm{pl}$ & vh & friable & . \\
\hline & & $\begin{array}{l}\mathrm{Cl} \\
2 \mathrm{C} 2\end{array}$ & $\begin{array}{l}0-15 \\
0 .-25\end{array}$ & $\begin{array}{l}7.5 \mathrm{YR} \\
7.5 \mathrm{YR} \\
-\end{array}$ & $5 / 6$ & 年/4 & : & $\begin{array}{l}\text { SL } \\
\text { LS }\end{array}$ & 1 & $f$ & sl & so & $\begin{array}{l}\text { Loose } \\
\text { Vffiche }\end{array}$ & as \\
\hline 7 & 198 & $2 \mathrm{C} 3$ & $25-40$ & $7.5 \mathrm{YR}$ & $5 / 6$ & $\begin{array}{r}4 / 4 \\
4 / 4\end{array}$ & : & LS & $\frac{1}{2}$ & $\mathrm{~m}$ & $\begin{array}{l}\text { pli } \\
\text { sbk }\end{array}$ & sim & 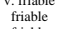 & aw \\
\hline & & $2 \mathrm{C} 4$ & $40-90$ & 10YR & $8 / 4$ & $6 / 6$ & - & LS & 2 & $\mathrm{~m}$ & sbk & $\mathrm{h}$ & friable & \\
\hline 8 & 205 & 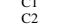 & $\begin{array}{l}0-20 \\
20-90\end{array}$ & $\begin{array}{l}5 Y R \\
5 Y R\end{array}$ & $\begin{array}{l}6 / 6 \\
7 / 6\end{array}$ & $\begin{array}{l}5 / 6 \\
6 / 6\end{array}$ & : & $\begin{array}{l}\text { LS } \\
\text { LS }\end{array}$ & $\frac{1}{2}$ & 孚 & $\begin{array}{c}\mathrm{pl} \\
\mathrm{sbk}\end{array}$ & $\begin{array}{l}\text { sh } \\
\text { h }\end{array}$ & $\begin{array}{l}\text { loose } \\
\text { friable }\end{array}$ & as \\
\hline & & & & & $7 / 4$ & $5 / 6$ & 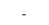 & LS & & 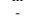 & & so & & as \\
\hline 9 & 208 & $2 \mathrm{C} 2$ & $20-50$ & $7.5 \mathrm{YR}$ & $5 / 8$ & $5 / 6$ & - & SL & 2 & $\mathrm{~m}$ & sbk & $\mathrm{h}$ & friable & as \\
\hline & & $2 \mathrm{C} 3$ & & 7.5YR & $7 / 6$ & $5 / 8$ & & SL & & $\mathrm{m}$ & stk & vh & friable & \\
\hline 10 & 198 & $\begin{array}{ll}\mathrm{Cl} \\
\mathrm{Cl}_{2}\end{array}$ & $\begin{array}{c}0-25 \\
25-6\end{array}$ & $\begin{array}{l}7.5 Y R \\
75.5 Y R\end{array}$ & $\begin{array}{l}7 / 6 \\
7 / 6\end{array}$ & 5 & - & $\begin{array}{l}S_{S L} \\
S L\end{array}$ & 2 & ${ }_{m}^{f}$ & $\begin{array}{c}\mathrm{pl} \\
\mathrm{spk}\end{array}$ & slh & $\begin{array}{l}\text { loose } \\
\text { firable }\end{array}$ & as \\
\hline 11 & 195 & C1 & $\begin{array}{c}29-60 \\
0-30\end{array}$ & 10YR & $8 / 4$ & 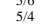 & few & $\begin{array}{l}\text { SL } \\
\text { LS }\end{array}$ & &. & sl & $\begin{array}{l}\text { h } \\
\text { so }\end{array}$ & $\begin{array}{l}\text { Trable } \\
\text { loose }\end{array}$ & as \\
\hline 11 & 193 & $\mathrm{C} 2$ & 30-100 & $7.5 \mathrm{YR}$ & $7 / 6$ & $5 / 6$ & & LS & 2 & $f$ & $\mathrm{pl}$ & $\mathrm{h}$ & friable & \\
\hline
\end{tabular}

\begin{tabular}{|c|c|c|c|c|c|c|c|c|c|c|c|c|c|c|}
\hline \multirow{2}{*}{$\begin{array}{l}\text { Prof. } \\
\text { No }\end{array}$} & \multirow{2}{*}{$\begin{array}{l}\text { Elevation } \\
\text { A.S. }(\mathrm{m})\end{array}$} & \multirow{2}{*}{ Horizon } & \multirow{2}{*}{$\begin{array}{l}\text { Depth } \\
(\mathrm{cm})\end{array}$} & \multicolumn{3}{|c|}{ Soil Color } & \multirow{2}{*}{ Gravel } & \multirow{2}{*}{$\begin{array}{l}\text { Texture } \\
\text { (I) }\end{array}$} & \multicolumn{3}{|c|}{ Soil Structure (II) } & \multicolumn{2}{|c|}{ Consistence (III) } & \multirow{2}{*}{$\begin{array}{c}\text { Boundary } \\
\text { (IV) }\end{array}$} \\
\hline & & & & Hue & & Moist & & & & & Type & & Moist & \\
\hline 12 & 193 & $\begin{array}{c}\mathrm{C} 1 \\
2 \mathrm{C} 2\end{array}$ & $\begin{array}{c}0-30 \\
30-70\end{array}$ & $\begin{array}{l}7.5 \mathrm{YR} \\
10 \mathrm{YR}\end{array}$ & $\begin{array}{l}7 / 4 \\
8 / 4\end{array}$ & $\begin{array}{l}5 / 6 \\
7 / 4\end{array}$ & few & $\begin{array}{l}\text { SL } \\
\text { LS }\end{array}$ & 2 & f & $\begin{array}{c}\mathrm{sl} \\
\mathrm{sl} \\
\mathrm{sbk}\end{array}$ & $\begin{array}{c}\text { so } \\
\text { h }\end{array}$ & $\begin{array}{c}\text { loose } \\
\text { v. friable }\end{array}$ & $\begin{array}{l}\text { as } \\
\text { aw }\end{array}$ \\
\hline & & & $70-100$ & $5 \mathrm{YR}$ & & $5 / 6$ & & SL & & $\mathrm{m}$ & & vh & friable & ${ }^{2}$ \\
\hline 13 & 198 & $c_{1}$ & $\begin{array}{l}0.30 \\
30.80\end{array}$ & (10k & 要4 & 列 & $\therefore$ & 列 & 2 & 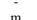 & st & 空o & $\begin{array}{l}\text { losese } \\
\text { froble }\end{array}$ & as \\
\hline & & & & & $4 / 8$ & $4 / 4$ & & SL & & & & so & & \\
\hline 14 & 200 & $2 \mathrm{C} 2$ & $30-60$ & $7.5 \mathrm{YR}$ & $6 / 4$ & $4 / 4$ & & LS & 1 & $f$ & pl & h & $\begin{array}{l}\text { Triable } \\
\text { frose }\end{array}$ & as \\
\hline & & $2 \mathrm{C} 3$ & $60-80$ & $\begin{array}{l}7.5 \mathrm{YR} \\
7.5 \mathrm{YR}\end{array}$ & $\begin{array}{l}6 / 4 \\
7 / 4\end{array}$ & $\begin{array}{l}4 / 4 \\
5 / 6\end{array}$ & & LS & 2 & $\mathrm{~m}$ & sbk & $\begin{array}{c}\text { vh } \\
\text { so }\end{array}$ & $\begin{array}{l}\text { friable } \\
\text { loose }\end{array}$ & as \\
\hline 15 & 197 & $\mathrm{C}_{2}$ & $15-30$ & $7.5 \mathrm{YR}$ & $6 / 4$ & $5 / 6$ & : & LS & 1 & f & 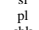 & slh & 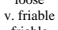 & as \\
\hline & & $2 \mathrm{C} 3$ & $30-70$ & $\begin{array}{l}7.5 \mathrm{YR} \\
10 \mathrm{R}\end{array}$ & $\begin{array}{l}6 / 6 \\
5 / 6 \\
6\end{array}$ & $5 / 6$ & & SL & & $\mathrm{m}$ & sbk & $\mathrm{h}$ & $\begin{array}{l}\text { friable } \\
\text { floses }\end{array}$ & \\
\hline 16 & 196 & $\mathrm{C}_{2}$ & $10-50$ & $\begin{array}{l}10 \mathrm{YR} \\
10 \mathrm{R}\end{array}$ & 5/6 & 4/4 & - & LS & 1 & f & pl & $\begin{array}{l}\text { so } \\
\text { sh }\end{array}$ & $\begin{array}{l}\text { 年osce } \\
\text { v. friable }\end{array}$ & as \\
\hline & & $2 \mathrm{C} 3$ & $50-100$ & $10 \mathrm{YR}$ & $5 / 3$ & $4 / 3$ & & s & 2 & co & $\mathrm{pl}$ & $\mathrm{h}$ & friable & \\
\hline & & $\mathrm{C} 1$ & & 7.5YR & $7 / 4$ & $5 / 6$ & few & SL & 1 & & sl & so & loose & as \\
\hline 17 & 195 & $\mathrm{C}_{\mathrm{C}}$ & 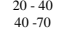 & $\begin{array}{l}10 \mathrm{YR} \\
10 \mathrm{R}\end{array}$ & $8 / 2$ & $5 / 6$ & & $\begin{array}{l}\text { SL } \\
\text { SL }\end{array}$ & $\begin{array}{l}1 \\
2\end{array}$ & $\begin{array}{l}\mathrm{m} \\
\mathrm{m}\end{array}$ & $\begin{array}{c}\mathrm{pl} \\
\mathrm{pl}\end{array}$ & $\begin{array}{c}\text { h } \\
\text { exh }\end{array}$ & $\begin{array}{l}\text { friable } \\
\text { friable }\end{array}$ & as \\
\hline 18 & 194 & C1 & & ${ }_{5 Y R} \mathrm{YR}$ & $6 / 6$ & $5 / 6$ & few & LS & 2 & & $\mathrm{pl}$ & slh & loose & as \\
\hline & & $\begin{array}{c}\mathrm{C} 2 \\
2 \mathrm{C} 3\end{array}$ & $\begin{array}{l}15-35 \\
35-80\end{array}$ & $\begin{array}{l}5 \mathrm{YR} \\
7.5 \mathrm{YR}\end{array}$ & $\begin{array}{l}7 / 6 \\
6 / 6\end{array}$ & $5 / 6$ & - & $\begin{array}{l}\text { LS } \\
\text { SL }\end{array}$ & $\frac{2}{2}$ & $\begin{array}{l}\mathrm{m} \\
\mathrm{m}\end{array}$ & bk & $\begin{array}{c}\mathrm{h} \\
\mathrm{yh}\end{array}$ & $\begin{array}{l}\text { loose } \\
\text { v friable }\end{array}$ & as \\
\hline 19 & 198 & C1 & & $7.5 \mathrm{YR}$ & $7 / 6$ & $5 / 6$ & & LS & 1 & $\mathrm{f}$ & $\mathrm{pl}$ & slh & loose & as \\
\hline & & ${ }^{2 \mathrm{C} 2} 2$ & 30- & & $\begin{array}{l}7 / 6 \\
7 / 6\end{array}$ & $5 / 6$ & few & $\begin{array}{l}\text { SL } \\
\text { LS }\end{array}$ & $2^{2}$ & $\mathrm{~m}$ & sbk & $\begin{array}{c}\mathrm{h} \\
\mathrm{so}\end{array}$ & $\begin{array}{l}\text { v. friable } \\
\text { lose }\end{array}$ & \\
\hline 20 & 196 & $2 \mathrm{C} 2$ & $20-50$ & $7.5 Y \mathrm{YR}$ & $6 / 6$ & $5 / 6$ & & SL & 1 & $\mathrm{f}$ & sbk & slh & v. friable & aw \\
\hline & & & & 7.5YR & & & & SL & & & & & frriable & \\
\hline
\end{tabular}

Abbreviations:

Texture (1): $S=$ Sand, $\mathrm{LS}=$ Loamy Sand and $\mathrm{SL}=$ Sandy Loam Soil structure (II): 1 = weak, $2=$ moderate, $3=$ strong, $\mathrm{f}=$ fine $\mathrm{m}=$ medium, $\mathrm{co}=$ coarse, $\mathrm{sl}=$ structureless, $\mathrm{pl}=$ platy and sbk= subangular blocky. Consistence (III): so = soft, sh = slightly hard, $\mathrm{h}=\mathrm{hard}, \mathrm{vh}=$ very hard, and exh $=$ extremely hard

Boundary (IV): as = abrupt smooth, and aw = abrupt wavy.

\subsection{Main Physical and Chemical Properties of The Studied Soils}

The main physical and chemical properties are given in Tables 2 and 3, respectively, and are illustrated in Figure 2. These results showed that the soil profiles were generally medium deep to deep and the soil texture was mainly coarse (sand, loamy sand and sandy loam). The calcium carbonate content ranged from 0.92 to $12.60 \%$ with a general trend to decrease with depth. The results also displayed that the gypsum content was very low $(<0.5 \%)$. Soil reaction was mildly to moderately alkaline as indicated by $\mathrm{pH}$ values, which ranged between 7.6 and 8.1. In some cases, $\mathrm{pH}$ values of the surface layers were considerably higher than those of the subsurface ones. This pattern was conversely correlated with the concentration of total soluble salts. The soils of the study area were nonto slightly saline as the ECe values varied between 0.53 and $6.85 \mathrm{dSm}-1$, except in few soil samples that they were considered moderately saline as the ECe extended from 8.17 to $11.37 \mathrm{dSm}-1$.

Table 2: Some physical properties of soils, as well as their taxa, of the studied profiles

\begin{tabular}{|c|c|c|c|c|c|}
\hline $\begin{array}{l}\text { Prof. } \\
\text { No. }\end{array}$ & $\begin{array}{c}\text { Depth of } \\
\text { Layer }(\mathrm{cm})\end{array}$ & $\begin{array}{c}\mathrm{CaCO}_{3} \\
\%\end{array}$ & $\begin{array}{c}\text { Gypsum } \\
\%\end{array}$ & $\begin{array}{l}\text { Soli Texture } \\
\text { Grade }\end{array}$ & Classification \\
\hline \multirow{2}{*}{1} & $0-20$ & 3.36 & 0.04 & Loamy Sand & \multirow{2}{*}{ Typic Torriorthents } \\
\hline & $20-100$ & 2.94 & 0.06 & Sandy Loam & \\
\hline \multirow{3}{*}{2} & $0-25$ & 6.32 & 0.05 & Loamy Sand & \multirow{3}{*}{ Typic Torripsamments } \\
\hline & $25-50$ & 2.10 & 0.05 & Sand & \\
\hline & $50-100$ & 1.76 & 0.05 & Sand & \\
\hline \multirow{2}{*}{3} & $0-15$ & 10.5 & 0.10 & Sand & \multirow{2}{*}{ Typic Torripsamments } \\
\hline & $15-70$ & 1.51 & 0.08 & Loamy Sand & \\
\hline \multirow{2}{*}{4} & $0-20$ & 7.14 & 0.06 & Sandy Loam & \multirow{2}{*}{ Lithic Torriorthents } \\
\hline & $20-50$ & 2.18 & 0.07 & Sandy Loam & \\
\hline \multirow{3}{*}{5} & $0-15$ & 2.02 & 0.36 & Sandy Loam & \multirow{3}{*}{ Typic Torriorthents } \\
\hline & $15-30$ & 2.18 & 0.13 & Loamy Sand & \\
\hline & $30-90$ & 2.10 & 0.18 & Sandy Loam & \\
\hline \multirow{3}{*}{6} & $0-20$ & 10.92 & 0.06 & Sandy Loam & \multirow{3}{*}{ Typic Torriorthents } \\
\hline & $20-50$ & 3.44 & 0.07 & Sandy Loam & \\
\hline & $50-100$ & 5.54 & 0.02 & Sandy Loam & \\
\hline
\end{tabular}


Malaysian Journal of Sustainable Agriculture (MJSA) 2(2) (2018) 09-15

\begin{tabular}{|c|c|c|c|c|c|}
\hline \multirow{2}{*}{7} & $0-15$ & 1.85 & 0.07 & Sandy Loam & \multirow{2}{*}{ Typic Torripsamments } \\
\hline & $15-25$ & 2.02 & 0.05 & Loamy Sand & \\
\hline & $25-40$ & 1.01 & 0.06 & Loamy Sand & \\
\hline & $40-90$ & 9.32 & 0.05 & Loamy Sand & \\
\hline \multirow{2}{*}{8} & $0-20$ & 5.04 & 0.06 & Loamy Sand & \multirow{2}{*}{ Typic Torripsamment } \\
\hline & $20-90$ & 1.68 & 0.15 & Loamy Sand & \\
\hline \multirow{3}{*}{9} & $0-20$ & 3.11 & 0.07 & Loamy Sand & \multirow{3}{*}{ Typic Torriorthents } \\
\hline & $20-50$ & 2.52 & 0.11 & Sandy Loam & \\
\hline & $50-80$ & 9.41 & 0.06 & Sandy Loam & \\
\hline \multirow{2}{*}{10} & $0-25$ & 2.10 & 0.05 & Sandy Loam & \multirow{2}{*}{ Typic Torriorthents } \\
\hline & $25-60$ & 2.94 & 0.04 & Sandy Loam & \\
\hline \multirow{2}{*}{11} & $0-30$ & 3.28 & 0.02 & Loamy Sand & \multirow{2}{*}{ Typic Torripsamments } \\
\hline & $30-100$ & 1.93 & 0.03 & Loamy Sand & \\
\hline \multirow{3}{*}{12} & $0-30$ & 3.86 & 0.06 & Sandy Loam & \multirow{3}{*}{ Typic Torripsamments } \\
\hline & $30-70$ & 1.76 & 0.07 & Loamy Sand & \\
\hline & $70-100$ & 0.92 & 0.07 & Sandy Loam & \\
\hline \multirow{2}{*}{13} & $0-30$ & 11.0 & 0.12 & Sandy Loam & \multirow{2}{*}{ Typic Torriorthents } \\
\hline & $30-80$ & 9.66 & 0.03 & Sandy Loam & \\
\hline \multirow{3}{*}{14} & $0-30$ & 3.78 & 0.06 & Sandy Loam & \multirow{3}{*}{ Typic Torripsamments } \\
\hline & $30-60$ & 1.26 & 0.04 & Loamy Sand & \\
\hline & $60-80$ & 1.60 & 0.01 & Loamy Sand & \\
\hline \multirow{3}{*}{15} & $0-15$ & 7.14 & 0.04 & Loamy Sand & \multirow{3}{*}{ Typic Torripsamments } \\
\hline & $15-30$ & 4.37 & 0.05 & Loamy Sand & \\
\hline & $30-70$ & 4.79 & 0.04 & Sandy Loam & \\
\hline \multirow{3}{*}{16} & $0-10$ & 3.53 & 0.06 & Loamy Sand & \multirow{3}{*}{ Typic Torripsamments } \\
\hline & $10-50$ & 1.26 & 0.06 & Loamy Sand & \\
\hline & $50-100$ & 1.09 & 0.03 & Sand & \\
\hline \multirow{3}{*}{17} & $0-20$ & 9.66 & 0.09 & Sandy Loam & \multirow{3}{*}{ Typic Torriorthents } \\
\hline & $20-40$ & 3.36 & 0.19 & Sandy Loam & \\
\hline & $40-70$ & 1.26 & 0.08 & Sandy Loam & \\
\hline \multirow{3}{*}{18} & $0-15$ & 9.24 & 0.10 & Loamy Sand & \multirow{3}{*}{ Typic Torriorthents } \\
\hline & $15-35$ & 5.46 & .040 & Loamy Sand & \\
\hline & $35-80$ & 10.16 & 0.09 & Sandy Loam & \\
\hline \multirow{2}{*}{19} & $0-30$ & 5.12 & 0.03 & Loamy Sand & \multirow{2}{*}{ Typic Torripsamments } \\
\hline & $30-60$ & 3.44 & 0.18 & Sandy Loam & \\
\hline \multirow{3}{*}{20} & $0-20$ & 4.20 & 0.09 & Sandy Loam & \\
\hline & $20-50$ & 5.46 & 0.05 & Sandy Loam & Typic Torriorthents \\
\hline & $50-80$ & 12.60 & 0.06 & Sandy Loam & \\
\hline
\end{tabular}

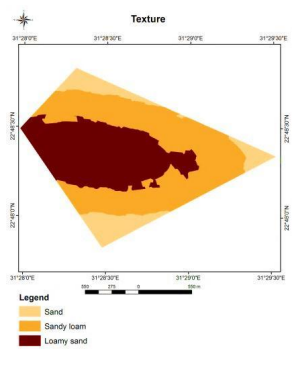

Soil texture map

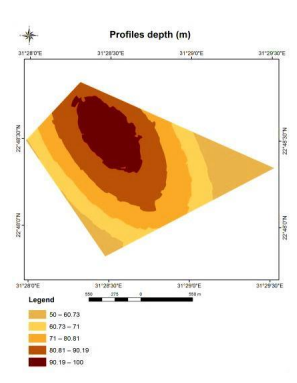

Soil depth map

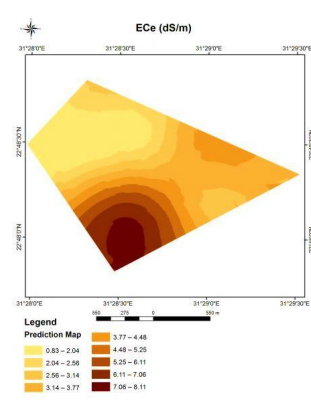

Soil salinity (ECe) map

The low values of electrical conductivity (ECe) may be due to free drainage conditions. Most of soil profiles showed a clear increase in the soluble salts with depth. Moreover, these soils exhibited no sodicity as they had exchangeable sodium percentage (ESP) values that were less than $15 \%$ and sodium adsorption ratio (SAR) values which were less than 13 , except the subsurface layer of profile 18 that showed a SAR value that was higher than 13 (18.11). The examined soil samples displayed that the cation exchange capacity (CEC) was very low $(5.11-14.65 \mathrm{cmol}(+) / \mathrm{kg})$ due to their coarse texture and their extremely low content of organic matter due to the prevailing arid climate and barren nature of the soils [6]. The predominant climate of the study area was extremely arid, and the dominant soil moisture regime was aridic (torric) with a hyperthermic soil temperature regime. The investigated soils are classified according to Soil Survey Staff (2014) as Typic Torripsamments, Typic Torriorthents and Lithic Torriorthents (Table 2 and Figure 3).

Table 3: Some chemical properties of studied soil profiles

\begin{tabular}{|ccccccc|}
\hline $\begin{array}{c}\text { Prof. } \\
\text { No. }\end{array}$ & $\begin{array}{c}\text { Depth of } \\
\text { Layer }(\mathrm{cm})\end{array}$ & $\begin{array}{c}\text { PH } \\
(1: 1)\end{array}$ & $\begin{array}{c}\mathrm{EC}_{\mathrm{e}} \\
\left(\mathrm{dSm}^{-1}\right)\end{array}$ & $\begin{array}{c}\text { ESP } \\
\%\end{array}$ & SAR & $\begin{array}{c}\text { CEC } \\
\mathrm{cmol}(+) / \mathrm{kg}\end{array}$ \\
\hline \multirow{2}{*}{1} & $0-20$ & 7.9 & 3.70 & 2.98 & 4.56 & 9.22 \\
& $20-100$ & 8.0 & 3.33 & 3.97 & 3.93 & 8.51 \\
\hline \multirow{2}{*}{2} & $0-25$ & 7.7 & 1.58 & 2.01 & 3.33 & 9.21 \\
& $25-50$ & 7.6 & 0.66 & 0.86 & 1.20 & 7.11 \\
& $50-100$ & 7.9 & 0.53 & 0.54 & 1.38 & 7.35 \\
\hline \multirow{2}{*}{3} & $0-15$ & 7.7 & 1.42 & 2.47 & 3.99 & 6.42 \\
& $15-70$ & 7.7 & 3.50 & 11.99 & 9.99 & 5.21 \\
\hline \multirow{2}{*}{4} & $0-20$ & 7.9 & 2.94 & 5.09 & 4.38 & 13.12 \\
& $20-50$ & 7.7 & 6.12 & 4.17 & 5.21 & 10.24 \\
\hline
\end{tabular}

\begin{tabular}{|c|c|c|c|c|c|c|}
\hline \multirow{3}{*}{5} & $0-15$ & 7.67 & 3.73 & 7.43 & 8.09 & 6.61 \\
\hline & $15-30$ & 7.6 & 5.10 & 5.60 & 8.71 & 8.93 \\
\hline & $30-90$ & 8.1 & 2.13 & 4.11 & 4.61 & 9.32 \\
\hline \multirow{3}{*}{6} & $0-20$ & 7.8 & 1.46 & 3.72 & 3.0 & 12.51 \\
\hline & $20-50$ & 7.6 & 4.74 & 6.76 & 11.26 & 13.22 \\
\hline & $50-100$ & 7.6 & 3.95 & 2.72 & 2.32 & 14.65 \\
\hline \multirow{4}{*}{7} & $0-15$ & 8.0 & 5.01 & 6.67 & 5.78 & 10.52 \\
\hline & $15-25$ & 7.9 & 1.72 & 6.38 & 4.13 & 7.34 \\
\hline & $25-40$ & 7.8 & 2.85 & 3.66 & 9.14 & 6.44 \\
\hline & $40-90$ & 7.8 & 1.50 & 1.38 & 2.52 & 5.82 \\
\hline \multirow{2}{*}{8} & $0-20$ & .81 & 2.22 & 1.56 & 3.24 & 8.21 \\
\hline & $20-90$ & 7.7 & 1.12 & 3.70 & 2.87 & 7.95 \\
\hline \multirow{3}{*}{9} & $0-20$ & 8.0 & 0.90 & 1.81 & 2.20 & 6.44 \\
\hline & $20-50$ & 8.1 & 0.75 & 6.26 & 2.31 & 13.47 \\
\hline & $50-80$ & 8.0 & 1.32 & 4.16 & 4.82 & 12.67 \\
\hline \multirow{2}{*}{10} & $0-25$ & 8.0 & 2.09 & 2.33 & 3.34 & 12.78 \\
\hline & $25-60$ & 7.9 & 2.24 & 5.21 & 5.90 & 10.36 \\
\hline \multirow{2}{*}{11} & $0-30$ & 7.9 & 2.49 & 3.50 & 5.05 & 7.41 \\
\hline & $30-100$ & .78 & 4.80 & 4.97 & 9.10 & 8.53 \\
\hline \multirow{3}{*}{12} & $0-30$ & 7.9 & 1.81 & 1.66 & 4.45 & 9.64 \\
\hline & $30-70$ & 8.0 & 0.70 & 1.33 & 3.05 & 5.23 \\
\hline & $70-100$ & 7.8 & 0.77 & 1.64 & 1.80 & 10.42 \\
\hline \multirow{2}{*}{13} & $0-30$ & 7.7 & 1.78 & 1.98 & 3.37 & 5.38 \\
\hline & $30-80$ & 7.8 & 1.52 & 6.23 & 6.00 & 7.22 \\
\hline \multirow{3}{*}{14} & $0-30$ & 7.9 & 3.57 & 3.21 & 7.66 & 7.64 \\
\hline & $30-60$ & 7.9 & 6.77 & 4.96 & 10.45 & 5.11 \\
\hline & $60-80$ & 7.9 & 5.94 & 2.72 & 6.86 & 8.46 \\
\hline \multirow{3}{*}{15} & $0-15$ & 7.7 & 1.96 & 2.53 & 3.05 & 8.59 \\
\hline & $15-30$ & 7.8 & 1.26 & 1.39 & 2.46 & 6.37 \\
\hline & $30-70$ & .78 & 9.50 & 6.25 & 2.69 & 10.65 \\
\hline \multirow{3}{*}{16} & $0-10$ & 7.8 & 3.34 & 1.99 & 3.78 & 8.11 \\
\hline & $10-50$ & 7.8 & 5.78 & 2.51 & 8.86 & 6.41 \\
\hline & $50-100$ & 8.0 & 3.37 & 2.20 & 5.61 & 5.24 \\
\hline \multirow{3}{*}{17} & $0-20$ & 7.9 & 2.57 & 3.09 & 4.22 & 9.12 \\
\hline & $20-40$ & 7.9 & 6.85 & 5.07 & 9.46 & 9.34 \\
\hline & $40-70$ & 8.0 & 11.00 & 6.51 & 2.29 & 7.58 \\
\hline \multirow{3}{*}{18} & $0-15$ & 8.1 & 1.40 & 1.93 & 3.17 & 6.22 \\
\hline & $15-35$ & 7.9 & 2.55 & 3.90 & 7.15 & 8.41 \\
\hline & $35-80$ & .79 & 6.75 & 10.48 & 18.11 & 8.67 \\
\hline \multirow{2}{*}{19} & $0-30$ & .77 & 2.41 & 1.73 & 5.11 & 5.33 \\
\hline & $30-60$ & 7.7 & 0.69 & 4.97 & 2.86 & 12.55 \\
\hline \multirow{3}{*}{20} & $0-20$ & 7.9 & 3.12 & 2.32 & 4.47 & 8.23 \\
\hline & $20-50$ & 7.9 & 8.17 & 7.03 & 11.05 & 7.95 \\
\hline & $50-80$ & 7.8 & 11.37 & 4.17 & 7.21 & 10.69 \\
\hline
\end{tabular}

Where:

$\mathrm{ESP}=$ Exchangeable Sodium Percent

SAR $=$ Sodium Adsorption Ratio

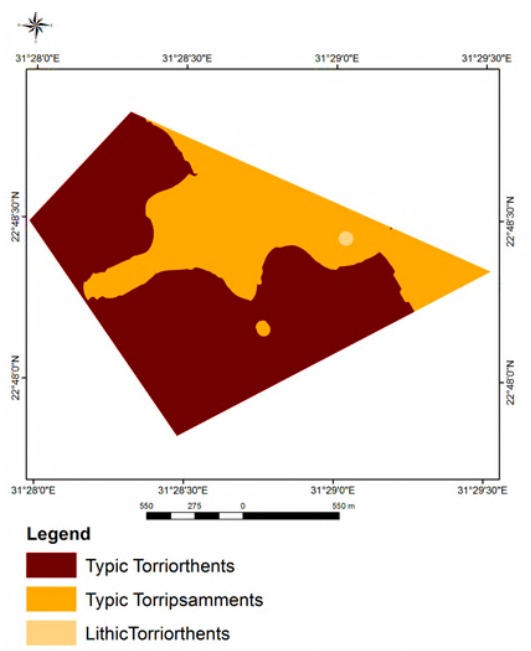

Figure 3: Soil classification map (subgroup level) of the study area

\subsection{Land Evaluation}

\subsubsection{Land Capability Classification}

Most of the land characteristics that were considered in the evaluation of the current land units under irrigation, ranged from very favorable to favorable for agricultural purposes. Qualitative land suitability studies were conducted using Modified Storie Index, MicroLEIS (Cervatana model) and Applied System of Land Evaluation (ASLE) program. Other information concerning climatic conditions and agricultural products were also used to predict the general land capability. From the agriculture point of view, 
soils of the study area are considered as promising soils. Evaluating their capability is an essential stage for the future practical use. Quantitative estimation of soil characteristics such as slope, drainage conditions (wetness), soil depth, texture, calcium carbonate content, gypsum status, salinity and sodicity were used in the land evaluation. The rating capability values and kinds of limitation condition types of the studied soils are present in Tables 4, 5 and 6 and illustrated in Figures 4. It is clear that none of the soil profiles was observed to be highly suitable (S1). It may be attributed to the slight or moderate limitations that are present in the study area. Accordingly, the study area could be classified into three classes as follow:

Class 2: This class includes the soils which are moderately suitable with a capability index (Ci) that varies between 60.85 and $63.68 \%$ (ASLE program) and good (Modified Storie Index). However, it disappears using MicroLEIS- CERVATANA model. It occupies 20 and $5 \%$ of the total area using ASLE program and Modified Storie Index, respectively. The soils of this class have slight limitations.

Class 3: This class contains the soils which have marginally suitable capability class C3 and capability index (Ci) that varies between 45.07 and $59.05 \%$ (ASLE program), fair (Modified Storie Index) and moderate (MicroLEIS- CERVATANA model). It occupies 80,50 and $100 \%$ of the study area using these respective land capability systems. The soils of this class are affected by moderate limitations.

Class 4: According to ASLE program this class comprises the soils which are not suitable for agricultural use, but they are suitable for pasture, have severe limitations that can be corrected and cover $45 \%$ of the study area. None of these land units was observed to be not suitable using Modified Storie Index and MicroLEIS- CERVATANA model [2, 22].

It could be concluded that the applied system of land evaluation (ASLE) is the most suitable program. It is preferable to be used as a qualitative land capability system for agricultural purposes. Compared to the other two programs, it is compatible with the Egyptian conditions. ASLE program can be also used by decision makers when they plan for future land utilization. The results of the current study indicated that the most limiting factors were soil texture followed by soil depth. Under good conditions of water availability for agricultural purposes, the moderately and marginally suitable soils (S2 and S3) could be safely used for agriculture.

Table 4: Land capability classes, grades and rating using ASLE program, MicroLEIS (Cervatana model) and Modified Storie Index

\begin{tabular}{|c|c|c|c|c|c|c|c|}
\hline \multicolumn{3}{|c|}{ ASLE Program } & \multicolumn{2}{|c|}{$\begin{array}{c}\text { MicroLEIS } \\
\text { (Cervatana } \\
\text { model) }\end{array}$} & \multicolumn{3}{|c|}{ Modified Storie Index } \\
\hline Class & Grade & $\begin{array}{l}\text { Rating } \\
(\%)\end{array}$ & Class & Grade & Class & Grade & $\begin{array}{l}\text { Rating } \\
(\%)\end{array}$ \\
\hline $\mathrm{C} 1$ & Excellent & $80-100$ & S1 & Excellent & Grade1 & Excellent & $80-100$ \\
\hline $\mathrm{C} 2$ & Good & $60-79$ & S2 & Good & Grade 2 & Good & $60-79$ \\
\hline $\mathrm{C} 3$ & Fair & $40-59$ & S3 & Moderate & Grade 3 & Fair & $40-59$ \\
\hline $\mathrm{C} 4$ & Poor & $20-39$ & $\mathrm{~N}$ & $\begin{array}{l}\text { Marginal } \\
\text { or Nil }\end{array}$ & Grade 4 & Poor & $20-39$ \\
\hline $\mathrm{C} 5$ & Very Poor & $10-19$ & -- & -- & Grade 5 & $\begin{array}{c}\text { Non } \\
\text { agricultural }\end{array}$ & $<20$ \\
\hline C6 & $\begin{array}{c}\text { Non } \\
\text { agricultural }\end{array}$ & $<10$ & -- & -- & -- & -- & -- \\
\hline
\end{tabular}

Table 5: Land capability classification of the studied soil profiles using ASLE, MicroLEIS and Modified Storie Index

\begin{tabular}{|cccccc|}
\hline \multirow{2}{*}{ Profile No. } & \multicolumn{3}{c}{ ASLE Program } & MicroLEIS (Cervatana model) & Modified Storie Index \\
\cline { 2 - 6 } & Class & $\%$ & Grade & Class & Class \\
\hline 1 & C3 & 55.38 & Fair & S3r & Grade 3 \\
2 & C3 & 57.22 & Fair & S3r & Grade 3 \\
3 & C3 & 45.07 & Fair & S3r & Grade 4 \\
4 & C3 & 52.84 & Fair & S3r & Grade 4 \\
5 & C3 & 59.05 & Fair & S3r & Grade 3 \\
6 & C2 & 63.68 & Good & S3r & Grade 3 \\
7 & C3 & 57.01 & Fair & S3r & Grade 3 \\
8 & C3 & 58.71 & Fair & S3r & Grade 3 \\
9 & C2 & 60.85 & Good & S3r & Grade 3 \\
10 & C2 & 61.55 & Good & S3r & Grade 4 \\
11 & C3 & 54.25 & Fair & S3r & Grade 3 \\
12 & C2 & 61.89 & Good & S3r & Grade 2 \\
13 & C3 & 52.65 & Fair & S3r & Grade 3 \\
14 & C3 & 52.71 & Fair & S3r & Grade 4 \\
15 & C3 & 49.23 & Fair & S3r & Grade 4 \\
16 & C3 & 55.2 & Fair & S3r & Grade 4 \\
17 & C3 & 51.22 & Fair & S3r & Grade 4 \\
18 & C3 & 55.01 & Fair & S3r & Grade 4 \\
19 & C3 & 50.27 & Fair & S3r & Grade 3 \\
20 & C3 & 50.14 & Fair & S3lr & Grade 4 \\
\hline
\end{tabular}

Table 6: Land capability classification of the study area according to ASLE Program, MicroLEIS (Cervatana model) and Modified Storie Indexf the studied soil profiles using ASLE, MicroLEIS and Modified Storie Index

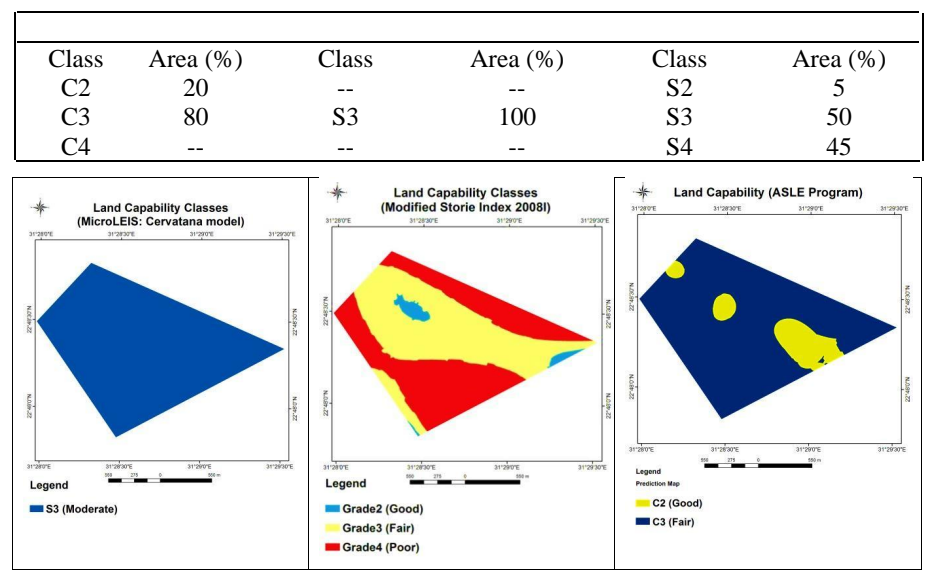

Figure 4: Land capability maps of the study area using different evaluation programs

The results of this research showed that $90 \%$ of total area was suitable for agricultural use. The area currently lacks soils of high capability for agricultural use. However, improving the soil properties and applying modern irrigation systems, the soil could be improved to be highly suitable for agricultural use. One of the best ways to improve such light soils (sandy soils) is through additions of organic materials. Good sources of organic matter include manures, leaf mold, sawdust, and straw. Many farmers enrich soils with natural fertilizers, such as animal manure, green manure, and compost. Continuous agriculture use of these soils will upgrade their suitability in the future.

\subsubsection{Soil Suitability Classification}

Land suitability assessment for agriculture is means to evaluate the ability of a piece of land to provide the optimal ecological requirements for a certain crop variety. In other words, it evaluates the capability of land in enabling optimum crop development and maximum productivity. This evaluation needs a specification of the respective crop requirements and calibrating them with the nature of the land and soil parameters. The current study used two programs, namely applied system of land evaluation (ASLE) and MicroLEIS (ALMAGRA model) which were used in the quantitative parameters of the agro-ecological evaluation in the study area for the land use types of different field crops. The studied soil profiles were evaluated to determine their suitability for growing different crops according to these two programs. The soil parameters used for estimating the suitability index for different crops were, climate, slope, drainage, texture, soil profile depth, calcium carbonate, gypsum status, $\mathrm{pH}$, salinity and sodicity.

The results indicate that the area under consideration has a good potential to produce field crops under irrigation, provided that the water requirements are met. Eleven crops were elected to assess their suitability for agriculture, namely alfalfa, wheat, maize, cotton, soybean, sunflower, sugar beet, watermelon, potato, citrus and olive. These crops are most suitable for arid and semi-arid soils (Tables 7, 8 and 9) and are illustrated in Figures 5, 6 and 7.

Table 7: Land suitability classes of the study area for different crops using the ASLE program

\begin{tabular}{|c|c|c|c|c|c|c|c|c|c|c|c|}
\hline \multirow{2}{*}{$\begin{array}{c}\text { Profile } \\
\text { No. }\end{array}$} & \multicolumn{11}{|c|}{ Soil Suitability (ASLE Program) } \\
\hline & Wheat & Maize & Watermelon & Potato & $\begin{array}{l}\text { Soya } \\
\text { bean }\end{array}$ & Cotton & Sunflower & $\begin{array}{c}\begin{array}{c}\text { Sugar } \\
\text { beet }\end{array}\end{array}$ & Alfalfa & Citrus & Olive \\
\hline 1 & S2 & S2 & S2 & S2 & S3 & S2 & S2 & S2 & S2 & S2 & S2 \\
\hline 2 & S2 & S2 & S2 & S2 & S2 & S2 & S2 & S2 & S2 & S2 & S2 \\
\hline 3 & S2 & S3 & S2 & S2 & S3 & S3 & S2 & S2 & S2 & NS1 & S4 \\
\hline 4 & S2 & S2 & S2 & S2 & NS1 & S4 & S4 & S2 & S2 & S4 & S4 \\
\hline 5 & S2 & S2 & S2 & S2 & S3 & S2 & S2 & S2 & S2 & S3 & S2 \\
\hline 6 & S2 & S2 & S2 & S2 & S2 & S2 & S2 & S2 & S2 & S2 & S1 \\
\hline 7 & S2 & S2 & S2 & S2 & S3 & S2 & S2 & S2 & S2 & S2 & S2 \\
\hline 8 & S2 & S2 & S2 & S2 & S3 & S2 & S2 & S2 & S2 & S2 & S2 \\
\hline 9 & S2 & S2 & S2 & S2 & S3 & S2 & S2 & S2 & S2 & S4 & S4 \\
\hline 10 & S2 & S2 & S2 & S2 & S3 & S2 & S2 & S2 & S2 & S4 & S4 \\
\hline 11 & S2 & S2 & S2 & S2 & S3 & S2 & S2 & S2 & S2 & S2 & S2 \\
\hline 12 & S2 & S2 & S1 & S2 & S2 & S2 & S2 & S2 & S2 & S2 & S2 \\
\hline 13 & S2 & S2 & S2 & S2 & S3 & s3 & S2 & S2 & S2 & NS1 & S4 \\
\hline 14 & S2 & S3 & S2 & S2 & S3 & S2 & S2 & S2 & S2 & NS1 & S4 \\
\hline 15 & S2 & S2 & S2 & S2 & S3 & S2 & S2 & S2 & S2 & S4 & S4 \\
\hline 16 & S2 & S2 & S2 & S2 & S3 & S2 & S2 & S2 & S2 & S2 & S2 \\
\hline 17 & S2 & S2 & S2 & S2 & S3 & S2 & S2 & S2 & S2 & NS1 & S4 \\
\hline 18 & S2 & S2 & S2 & S2 & S3 & S2 & S2 & S2 & S2 & S4 & S4 \\
\hline
\end{tabular}




\begin{tabular}{|c|c|c|c|c|c|c|c|c|c|c|c|}
\hline 19 & S2 & s2 & S2 & S2 & s3 & s3 & s2 & S2 & S2 & NS1 & S4 \\
\hline 20 & $\mathrm{~S} 2$ & S2 2 & S2 & $\mathrm{S} 2$ & S3 & S2 2 & S2 & $\mathrm{S} 2$ & S2 & NS1 & \\
\hline & $\begin{array}{l}1=h \\
4=n \\
\text { itab }\end{array}$ & y suit & & & $\begin{array}{l}\text { suital } \\
=\text { cur }\end{array}$ & & table & $\begin{array}{l}\text { S3 } \\
\text { NS }\end{array}$ & & ly suit: & \\
\hline
\end{tabular}

Table 8: Land suitability classes of the study area for different crops using MicroLEIS-Almagra model

\begin{tabular}{|c|c|c|c|c|c|c|c|c|c|c|c|}
\hline \multirow{2}{*}{$\begin{array}{c}\text { Profile } \\
\text { No. }\end{array}$} & \multicolumn{11}{|c|}{ Soil Suitability (MicroLEIS-ALMAGRA Model) } \\
\hline & Wheat & Maize & $\begin{array}{l}\text { Water } \\
\text { melon }\end{array}$ & Potato & $\begin{array}{l}\text { Soya } \\
\text { bean }\end{array}$ & Cotton & Sunflower & $\begin{array}{c}\text { Sugar } \\
\text { beet }\end{array}$ & Alfalfa & Citrus & Olive \\
\hline 1 & S3 & S3 & s3 & s3 & $\mathrm{s3}$ & S3 & S3 & s3 & S3 & S2 & S2 \\
\hline 2 & S4 & S4 & S4 & S4 & S4 & S4 & S4 & S4 & S4 & S3 & S2 \\
\hline 3 & S4 & S4 & S4 & S4 & S4 & S4 & S4 & S4 & S4 & s3 & S2 \\
\hline 4 & S3 & S3 & S3 & S3 & S3 & S3 & S3 & S3 & S3 & S3 & S3 \\
\hline 5 & S3 & S3 & S3 & S3 & S3 & S3 & S3 & S3 & S3 & S2 & S2 \\
\hline 6 & S3 & S3 & S3 & S3 & S3 & S3 & S3 & S3 & S3 & S2 & S2 \\
\hline 7 & S4 & S4 & S4 & S4 & S4 & S4 & S4 & S4 & S4 & S3 & S2 \\
\hline 8 & S4 & S4 & S4 & S4 & S4 & S4 & S4 & S4 & S4 & S3 & S2 \\
\hline 9 & S3 & S3 & S3 & S3 & S3 & S3 & S3 & S3 & S3 & S2 & S2 \\
\hline 10 & S3 & S3 & S3 & S3 & S3 & S3 & S3 & S3 & S3 & S3 & S3 \\
\hline 11 & S4 & S4 & S4 & S4 & S4 & S4 & S4 & S4 & S4 & S3 & S2 \\
\hline 12 & S3 & S3 & S3 & S3 & S3 & S3 & S3 & S3 & S3 & S2 & S2 \\
\hline 13 & S3 & S3 & S3 & S3 & S3 & S3 & S3 & S3 & S3 & S2 & S2 \\
\hline 14 & S4 & S4 & S4 & S4 & S4 & S4 & S4 & S4 & S4 & S3 & S3 \\
\hline 15 & S3 & S3 & S3 & S3 & S3 & S3 & S3 & S3 & S3 & S3 & S3 \\
\hline 16 & S4 & S4 & S4 & S4 & S4 & S4 & S4 & S4 & S4 & S3 & S2 \\
\hline 17 & S3 & S3 & S3 & S3 & S3 & S3 & S3 & S3 & S3 & S4 & S3 \\
\hline 18 & S3 & S3 & S3 & S3 & S3 & S3 & S3 & S3 & S3 & S3 & S3 \\
\hline 19 & S3 & s3 & S3 & S3 & S3 & S3 & S3 & S3 & S3 & S3 & S3 \\
\hline 20 & S3 & S3 & S3 & S3 & S3 & S3 & S3 & S3 & S3 & S4 & S3 \\
\hline & shly & table & & $\begin{array}{l}\mathrm{S} 2 \\
\mathrm{~N} S\end{array}$ & uital & tly $\mathrm{n}$ & iitable & $\begin{array}{l}\text { S3 } \\
\text { NS }\end{array}$ & $\begin{array}{l}\text { odera } \\
\text { erman }\end{array}$ & $\begin{array}{l}\text { ly suita } \\
\text { tt not }\end{array}$ & $\begin{array}{l}\text { le } \\
\text { itable }\end{array}$ \\
\hline
\end{tabular}

Table 9: Soil suitability rating and percentage for growing some crops according to ASLE program and MicroLEIS-Almagra model.

\begin{tabular}{|c|c|c|c|c|c|c|c|c|c|c|c|}
\hline \multicolumn{12}{|c|}{ ASLE program } \\
\hline 胥 & 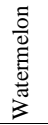 & 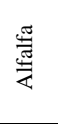 & $\frac{\vec{\Xi}}{5}$ & 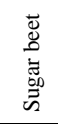 & 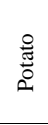 & 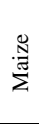 & 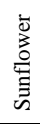 & : & $\stackrel{\Xi}{0}$ & 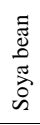 & 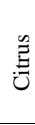 \\
\hline S1 & 5 & -- & -- & -- & -- & -- & -- & -- & 5 & -- & -- \\
\hline $\mathrm{S} 2$ & 95 & 100 & 100 & 100 & 100 & 90 & 95 & 80 & 40 & 15 & 40 \\
\hline S3 & -- & -- & -- & -- & -- & 10 & -- & 15 & -- & 80 & 5 \\
\hline S4 & -- & -- & -- & -- & -- & -- & 5 & 5 & 55 & -- & 25 \\
\hline NS1 & -- & -- & -- & -- & -- & -- & -- & -- & -- & 5 & 30 \\
\hline NS2 & -- & -- & -- & -- & -- & -- & -- & -- & -- & -- & -- \\
\hline \multicolumn{12}{|c|}{ MicroLEIS-Almagra model } \\
\hline 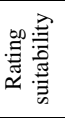 & $\stackrel{Ð}{\circlearrowright}$ & 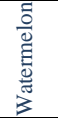 & 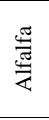 & $\begin{array}{l}\text { 芯 } \\
\stackrel{3}{\xi}\end{array}$ & 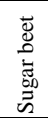 & 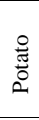 & 苋 & $\begin{array}{l}\overrightarrow{0} \\
\vdots \\
0 \\
0 \\
\vdots \\
\vdots \\
\omega\end{array}$ & 苞 & 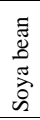 & 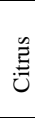 \\
\hline S1 & -- & -- & -- & -- & -- & -- & -- & -- & -- & -- & -- \\
\hline S2 & 60 & -- & -- & -- & -- & -- & -- & -- & -- & -- & 30 \\
\hline S3 & 40 & 65 & 65 & 65 & 65 & 65 & 65 & 65 & 65 & 65 & 60 \\
\hline S4 & -- & 35 & 35 & 35 & 35 & 35 & 35 & 35 & 35 & 35 & 10 \\
\hline NS1 & -- & -- & -- & -- & -- & -- & -- & -- & -- & -- & -- \\
\hline NS2 & -- & -- & -- & -- & -- & -- & -- & -- & -- & -- & -- \\
\hline $\mathrm{S} 1=\mathrm{h}$ & $1 \mathrm{~S}$ & & & $\mathrm{~S} 2=\mathrm{s}$ & ble & & & $3=$ & & abl & \\
\hline
\end{tabular}
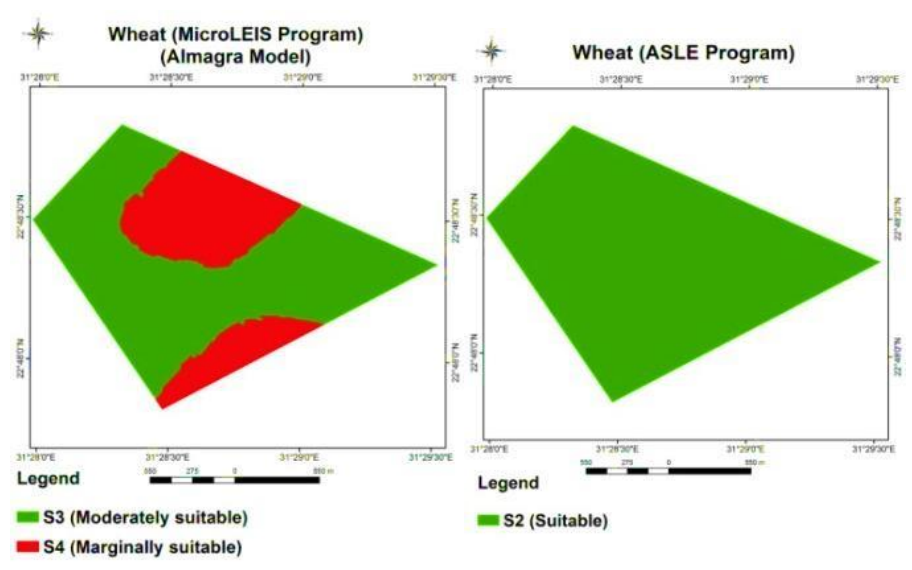
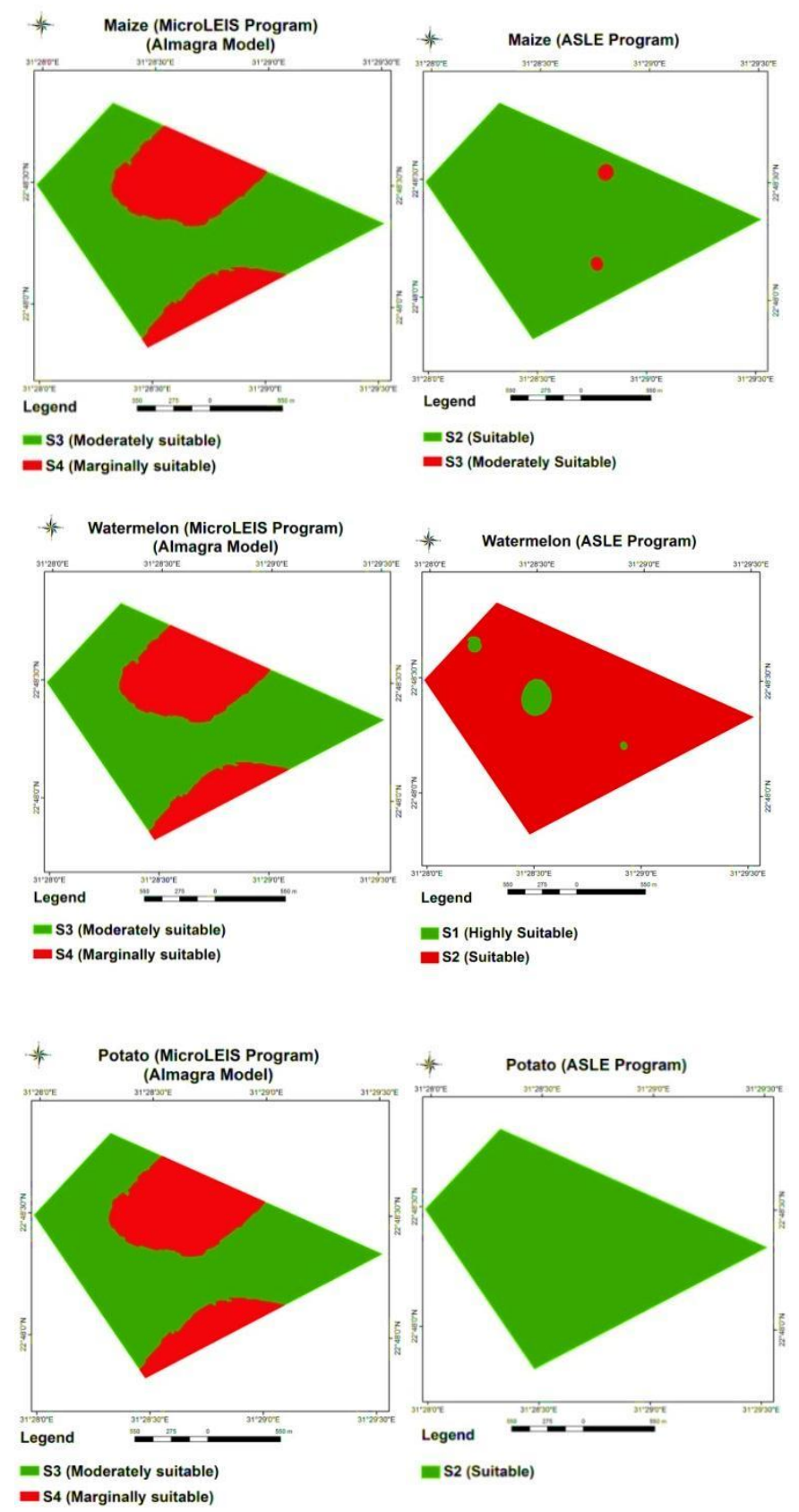

Figure 5: Land suitability maps for wheat, maize, watermelon and potato using ASLE and MicroLEIS (Almagr Model) programs
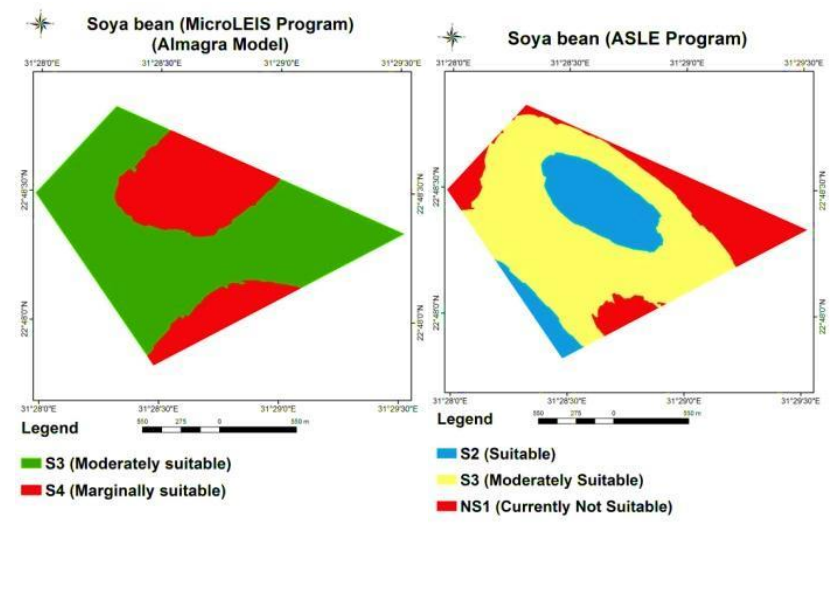

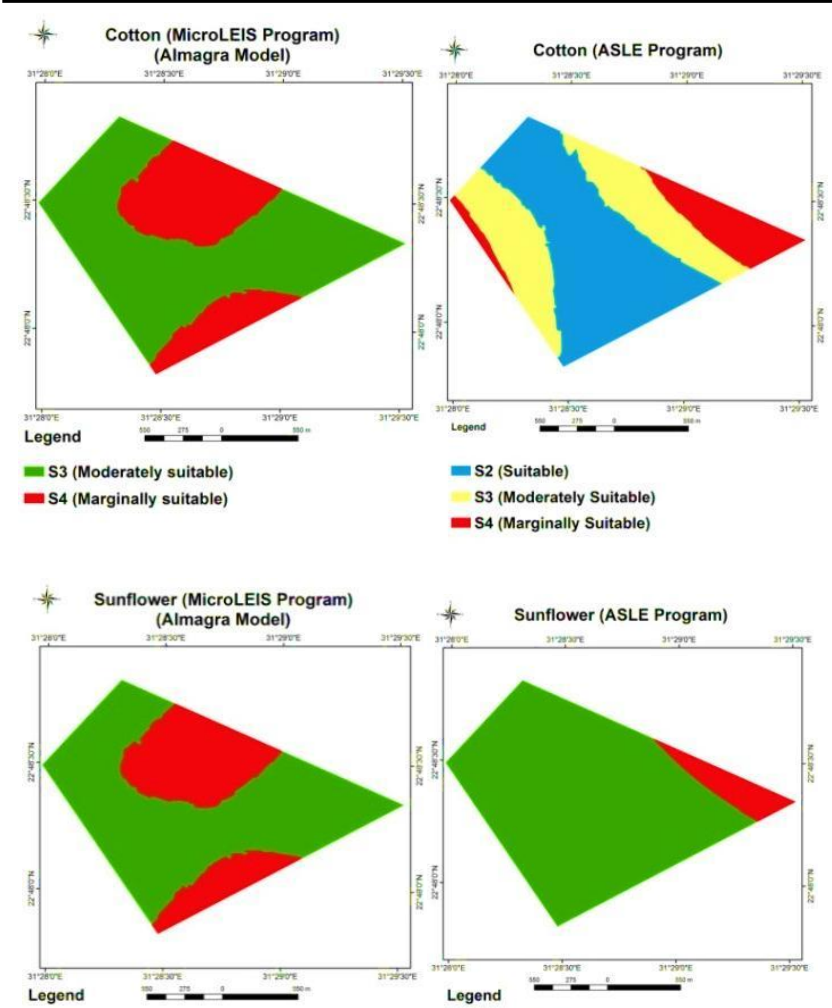

Legend

- $\$ 4$ (Marginally suitable)
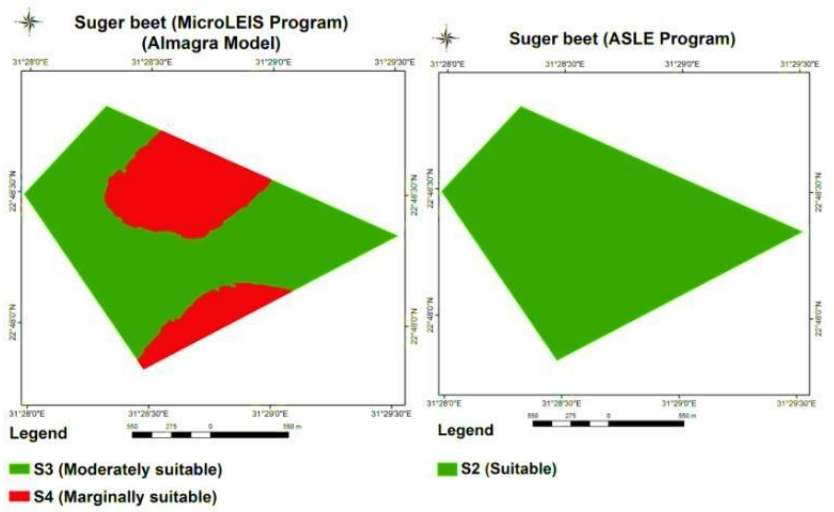

Figure 6: Land suitability maps for soybean, cotton, sunflower and sugar beet using ASLE and MicroLEIS (Almagr Model) programs

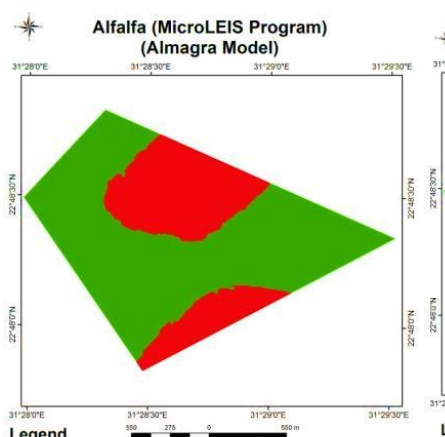

$$
\text { Legend }
$$

Es3 (Moderately suitable)

- S4 (Marginally suitable)

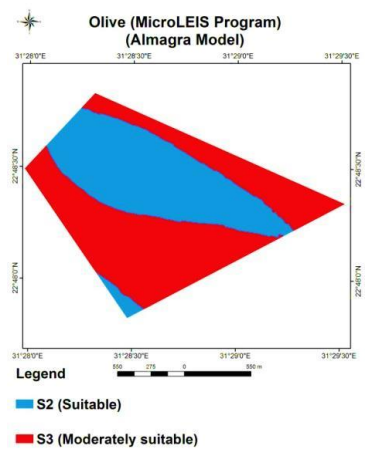

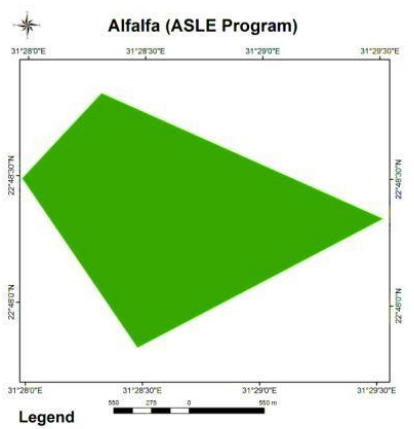

S2 (Suitable)

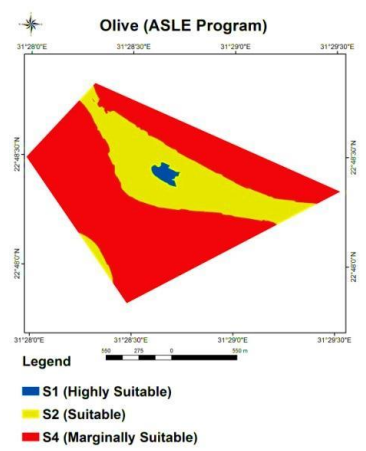

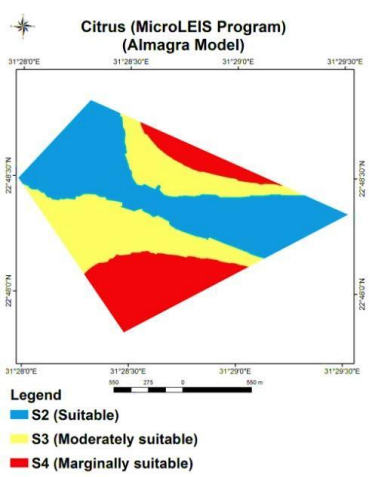

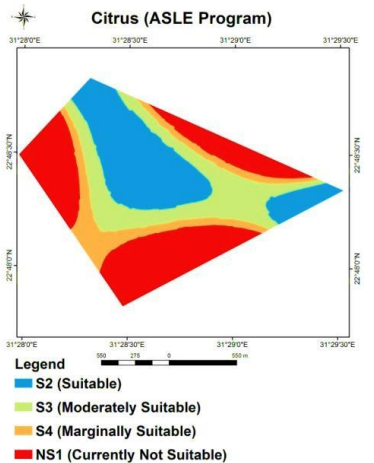

Figure 7: Land suitability maps for alfalfa, olive and citrus using ASLE and MicroLEIS (Almagr Model) programs

\subsubsection{Applied System of Land Evaluation (ASLE program)}

According to the applied system of land evaluation (ASLE program), the results indicated that $5 \%$ of the total study area are highly suitable (S1) and $95 \%$ are suitable (S2) for watermelon. All the study area (100\%) is suitable (S2) for alfalfa, wheat, sugar beet and potato. About $90 \%$ of the agricultural area are suitable and $10 \%$ are moderately suitable (S2) for maize. Most of the area $(95 \%)$ is suitable (S2) and $5 \%$ are marginally suitable (S4) for sunflower. For cotton cropping, $80 \%$ of the area are suitable, while $15 \%$ and $5 \%$ are moderately and marginally suitable, respectively. A small area (5\%) is highly suitable (S1), $40 \%$ are suitable and $55 \%$ are marginally suitable (S4) for growing olive. About 15 and $40 \%$ are suitable (S2), 80 and $5 \%$ are moderately suitable (S3), and 5 and $30 \%$ of the study area are not currently suitable (NS1) for soybean and citrus, respectively. Moreover, $25 \%$ of the total study area are marginally suitable (S4) for citrus cropping.

\subsubsection{MicroLEIS, ALMAGRA Model}

The current land suitability for different crops produced by MicroLEIS, ALMAGRA model showed that about $60 \%$ of the studied area are suitable (S2) and $40 \%$ are moderately suitable (S3) for olive. Crops such as: watermelon, alfalfa, wheat, sugar beet, potato maize, sunflower, cotton and soybean are moderately suitable (65\%) and marginally suitable $(35 \%)$ to be grown in this area (Tables 8 and 9 and Figures 5, 6 and 7). For growing citrus, about $30 \%$ of area are suitable, while $60 \%$ and $10 \%$ are moderately and marginally suitable, respectively $[5,14,20,22]$.

Some crops are considered unsuitable (NS1) due to the moderate to severe fertility limitations of the study area, soil depth and coarse texture. The coarse texture, shallow depth, and salinity of the soils in some soil profiles are the main limiting factors for growing crops especially fruit trees. Proper fertilization and management associated with intensive leaching can improve the soil suitability for growing various crops under consideration. Many options such as, use of crops which are categorized as suitable to the area can be raised for the sustainable use of the land for producing different crops. Correcting some limiting factors, such as $\mathrm{pH}$ through the application of organic fertilizers which can reduce the alkalinity of the soil and increase the soil organic matter through crop residue management are also options to increase the suitability of these soils for crop production.

\section{CONCLUSIONS}

The purpose of this study was to evaluate the soil capability and suitability of Tushka area for crop production and identify the factors that hinder the cultivation process. Agricultural land identification, according to its own ecological potentialities and limitations is a major objective of land use planning. This study implies a qualitative evaluation for the actual soil parameters to realize a precise and objective interpretation for the area under consideration and its suitability for a wide range of crops. The most effective soil parameters that influenced the land suitability of the study area were texture, soil depth and salinity. From applying different systems used for capability assessment (ASLE program, MicroLEIS and Modified Storie Index), most of the studied soils are good and moderately suitable for agriculture. The ASLE program was found to be suitable for the land suitability assessment for agricultural proposes of the study area. It is convenient to be used under Egyptian conditions. Also, it is more realistic for the application in arid and semi-arid areas. From the agriculture point of view, soils of the study area are considered as promising ones. Applying some corrections on the limiting soil factors, the potential capability of the soils will be improved. Some selected crops such as watermelon, alfalfa, wheat, sugar beet, potato maize, olive and sunflower are recommended to be grown in the study area. On the other hand, the soil maps produced for agricultural land suitability in this research can be helpful in carrying out the management processes. 


\section{REFERENCES}

[1] Bakeer, I.H.I. 2008. Using Geographic Information System (GIS) in Reassessment of Soil and Groundwater Salinity of Sohag Soils. M.Sc. Thesis, Fac. of Agric. Soil \& water Dept., Assiut Univ., Egypt.

[2] Sayed, A.S.A. 2013. Evaluation of the Land Resources for Agricultural Development - case study: El-Hammam Canal and its Extension, NW Coast of Egypt. Ph.D Thesis, Department Geowissenschaften, Universität Hamburg. Germany.

[3] Abdel-Hamid, M.A., Ismail, M., Nasr, Y.A., Kotb, Y. 2010. Assessment of Soils of Wadi El-Natrun Area, Egypt Using Remote Sensing and GIS Techniques. Journal of American Science, 10 (6),195 -206.

[4] Ismail, M., Nasr, Y.A., Kotb, Y. 2010. Assessment of Soils of Wadi ElNatrun Area, Egypt Using Remote Sensing and GIS Techniques. Journal of American Science, 6 (10), 195- 206.

[5] Aldabaa, A.A., Zhang, H., Shata, A., El-Sawey, S., Abdel-Hameed, A., Schroder, J.L. 2010. Land Suitability Classification of a Desert Area in Egypt for Some Crops Using Microleis Program. American-Eurasian Journal of Agriculture and Environment Science, 8 (1), 80-94.

[6] Abd El-Aziz, S.H. 2004. Characterization, Classification and Evaluation of Some Soils of Tushka, South Valley, Egypt. The Sec. Int. Conf. for Develop and the Environment in the Arab world, March 23-25. Assiut University Centre for Environmental Studies-Egypt.

[7] Sharififar, A. 2012. Assessment of different methods of soil suitability classification for wheat cultivation. Journal of Agrobiology, 29 (2), 47-54.

[8] Said, R. 1961. The Geology of Egypt. Elsevier Publishing Company, Amsterdam, New York, London.

[9] Khalifa, A.M. 2001. Chemical and mineralogical properties of some Toshka soils. M. Sc. Thesis, Cairo University, Egypt.

[10] Ismail, H.A., Morsy, I., El-Zahaby, E.M., El-Nagar, F.S. 2001. A developed expert system for land use planning by coupling land information system and modelling. Alexandria Journal of Agriculture Research, 46 (3), 141-154.

[11] Ageeb, G.W., Rahim, I.S., Wahba, M.M., Awad, F. 2004. Soil Potentialities as Useful Mean for Planning Agricultural Development in Sahl Baraka, Farafra Oasis, New Valley. II. Land Suitability Assessment for Some Crops. Egyptian Journal of Soil Science, 44 (2), 171-183.

[12] Higab, I.A. 2005. An evaluation of some soils of south El- Borolous lake Area. Ph. D. thesis, Fac. Agric Al-Azhar University, Egypt.

[13] Ismail, H.A., Bahnassy, M.H., Abd El-Kawy, O.R. 2005. Integrating GIS and modelling for agricultural land suitability evaluation at East Wadi ElNatroun Egypt. Egyptian Journal of Soil Science, 45 (3), 297-322.

[14] Darwish, Kh. M, Wahba, M.M., Awad, F. 2006. Agricultural Soil Suitability of Haplo-soils for Some Crops in Newly Reclamid Areas of Egypt. Journal of Applied Sciences Research, 2, 1235-1243.

[15] Shendi, M.M., Khater, E.A., Gomaa, O.R. 2006. Land Evaluation of Some Soils East of El-Fayoum Governorate, Adjacent to Assuit Desert Road. Agriculture and Food in Middle East, 3rd Egyptian-Syrian Conference 2006, El-Minia University, Egypt.

[16] Wahba, M.M., Darwish, Kh. M., Award, F. 2007. Suitability of Specific Crops Using MicroLEIS Program in Sahal Baraka, Farafra Oasis, Egypt. Journal of Applied Sciences Research, 3 (7), 531-539.

[17] Zamil, B.A., Abbd Allah, M.A.A., Abd El- Salam, G.M., El-Shahawy, M.I. 2009. Land evaluation of old irrigated soils in north delta region (Rewena area) at Kafr El Sheikh Governorate. Alexandria Science Exchange Journal, 30 (2), 214-228.

[18] Belal, A.B.A., Al-Ashri, K.M.A. 2011. GIS Based Land Evaluation in Baharyia Oasis, Western Desert, Egypt. Journal of Soil Science and Agriculture Engineering, Mansoura University, 2 (1), 11 -24.

[19] Ismail, M., Yacoub, R.K. 2012. Digital soil map using the capability of new technology in Sugar Beet area, Nubariya, Egypt. The Egyptian Journal of Remote Sensing and Space Sciences, 15, 113-124.

[20] Elsheikh, R., Abdul Rashid, B., Shariff, M., Amiri, F., Noordin, B.A., Balasundram, S. K., Mohd Soom, M.A. 2013. Agriculture Land Suitability
Evaluator (ALSE): A decision and planning support tool for tropical and subtropical crops. Computers and Electronics in Agriculture, 93, 98-110.

[21] Yousif, I.A.H. 2014. Land Evaluation and Sustainable Development of Some Areas of Dakhla Oasis, Egypt. Ph.D. Thesis, Faculty of Agriculture, Cairo University, Egypt.

[22] Ahmed, M.A.E. 2016. Land Evaluation of Gharb El-Mawhob Area, El Dakhla Oasis, New Valley, Egypt. M.Sc. Thesis, Faculty of Agriculture, Assiut University, Assiut, Egypt.

[23] Fanning, D.S., Fanning, M.C.B. 1989. Soil morphology, genesis and classification. John Wiley \& Sons. Inc., New York, USA.

[24] FAO. 2006. Guideline for soil description, fourth edition. Rome.

[25] Soil Survey Staff. 2014. Keys to Soil Taxonomy. Natural Resources Conservation Service, U.S. Dept. Agric., Twelfth Edition, U.S. Government printing office, Washington, D.C., USA.

[26] Soil Survey Staff. 1975. Soil Munsell Color Charts, USDA, Soil Conserve, Washington, DC.

[27] Schoenberger, P.J., Wysocki, D.A., Benham, E.C., Soil Survey Staff. 2012 Field Book for Describing and Sampling Soils, Version 3.0. Natural Resources Conservation Service, National Soil Survey Center, Lincoln, NE.

[28] Gavlak, R., Horneck, D., Miller, R.O. 2005. Soil, Plant and Water Reference Methods for the Western Region. Oregon State University Corvallis, OR. USA.

[29] Alvarenga, P., Palma, P., De Varennes, A., Cunha-Queda, A.C. 2012. A contribution towards the risk assessment of soils from the São Domingos Mine (Portugal): Chemical, microbial and ecotoxicological indicators. Environment Pollution, 161, 50- 56.

[30] Nelson, R.E. 1982. Carbonates and Gypsum. pp: 181-197. In: A. L. Page, R. H. Miller and D. R. Keeney (eds). Methods of Soil Analysis, Part 2. American Society of Agronomy, Inc., Madison, Wisconsin, USA.

[31] Houba, V.J.G., Van der Lee, J.J., Novozamsky, I. 1995. Soil analysis procedures: soil and plant analysis, part 5B. Department of Soil Science and Plant Nutrition, Wageningen Agriculture University, Wageningen.

[32] Bashour, I.I., Sayegh, A.H. 2007. Methods of Analysis for Soils of Arid and Semi-Arid Region, American University of Beirut, Lebanon. FAO, Rome.

[33] Rhoades, J.D. 1982. Cation exchange capacity. pp: 149-157. In: A. L. Page, R. H. Miller and D. R. Keeney (eds) Methods of Soil Analysis. Part 2. American Society of Agronomy, Inc., Madison, Wisconsin, USA.

[34] Lu, R. 2000. Soil and Agricultural Chemistry Analysis. Science and Technology of China Agriculture Press, Beijing, 1-336.

[35] O'Geen, A.T., Southard, S.B., Southard, R.J. 2008. A Revised Storie Index for Use with Digital Soils Information. University of California, Division of Agriculture and Natural Resources, USA.

[36] Aldbaa, A.A.A. 2012. Potential Land Use in the New Reclamation Areas at Wadi El-Rayan Depression and its Environs, EL-Fayum Governorate, Egypt. Ph.D. Thesis. Department of Soil Science, Faculty of Agriculture, Cairo University, Egypt.

[37] De la Rosa, D. 2000. MicroLEIS 2000: Conceptual Framework Agroecological land evaluation. Instituto de Recursos Naturalesy Agrobiologia, CSIC, Avda. Reina Mercedes 10, 41010 Sevilla, Spain, 267.

[38] Ismail, H., Morsi, I. 2001. Applied System of Land Evaluation (ASLE) in arid zones (software). Soil and water Science Department, Faculty of Agriculture, Alexandria University, Egypt.

[39] De la Rosa D., Mayol, F., Diaz-Pereira, E., Fernandez, M., De la Rosa, D. Jr. 2004. A land evaluation decision support system (MicroLEIS DSS) for agricultural soil protection with special reference to the Mediterranean region. Environmental Modelling and Software, 19, 929-942. 